\title{
Outpatient Treatment of Deep Vein Thrombosis in the United States: The Reasons for Geographic and Racial Differences in Stroke Study
}

\author{
Daniel Douce, MD¹, Leslie A. McClure, PhD, MS², Pamela Lutsey, PhD, MPH \\ Mary Cushman, MD, MSc ${ }^{1,4}$, Neil A. Zakai, MD, MSc ${ }^{1,4^{*}}$
}

\begin{abstract}
${ }^{1}$ Department of Medicine, Robert Larner College of Medicine, University of Vermont, Burlington, Vermont; 'Department of Epidemiology and Biostatistics, Dornsife School of Public Health, Drexel University, Philadelphia, Pennsylvania; 'Division of Epidemiology and Community Health, School of Public Health, University of Minnesota, Minneapolis, Minnesota; ${ }^{4}$ Department of Pathology and Laboratory Medicine, Robert Larner College of Medicine, University of Vermont, Burlington, Vermont.
\end{abstract}

BACKGROUND: Outpatient versus inpatient treatment of deep vein thrombosis (DVT) is believed to result in equivalent outcomes with decreased costs. Little is known about the adoption of outpatient DVT treatment in the United States.

OBJECTIVE: To describe the uptake of outpatient DVT treatment in the United States and understand how comorbidities and socioeconomic conditions impact the decision to treat as an outpatient.

DESIGN AND SETTING: The Reasons for Geographic and Racial Differences in Stroke cohort study recruited 30,329 participants between 2003 and 2007. DVT events were ascertained through 2011.

MEASUREMENTS: Multivariable logistic regression was used to determine the correlates of outpatient treatment of DVT accounting for age, sex, race, education, income, urban or rural residence, and region of residence.

RESULTS: Of 379 venous thromboembolism events, 141 participants had a DVT without diagnosed pulmonary embolism and that did not occur during hospitalization. Overall, $28 \%$ (39 of 141) of participants with DVT were treated as outpatients. In a multivariable model, the odds ratio for outpatient versus inpatient DVT treatment was 4.16 (95\% confidence interval [Cl], 1.25-13.79) for urban versus rural dwellers, $3.29(95 \% \mathrm{Cl}, 1.30-8.30)$ for white versus black patients, $2.41(95 \% \mathrm{Cl}, 1.06-5.47)$ for women versus men, and 1.90 (95\% Cl, 1.19-3.02) for every 10 years younger in age. Living outside the southeastern United States and having higher education and income were not statistically significantly associated with outpatient treatment.

CONCLUSIONS: Despite known safety and efficacy, only $28 \%$ of participants with DVT received outpatient treatment. This study highlights populations in which efforts could be made to reduce hospital admissions. Journal of Hospital Medicine 2017;12:826-830. Published online first September 6, 2017. (C) 2017 Society of Hospital Medicine
Venous thromboembolism (VTE) is a common medical condition comprising deep vein thrombosis (DVT) and pulmonary embolism (PE). Estimates of the incidence of DVT in the United States vary between 0.5 and 1.5 cases per 1000 person-years. ${ }^{1}$ Left untreated, roughly $50 \%$ of DVT patients progress to a PE, of whom $10 \%$ to $25 \%$ die within 3 months. ${ }^{2}$

Since the 1990s, multiple randomized controlled studies ${ }^{3-5}$ demonstrated the safety and efficacy of outpatient treatment for selected DVT patients with low molecular weight heparin and warfarin. The United States Food and Drug Administration approved enoxaparin, a low molecular weight heparin for outpatient use in $1998,{ }^{6}$ and by the end of the decade, multiple treatment guidelines for VTE acknowledged the safety of outpatient treatment of DVT with low molecular weight heparin in selected patients. ${ }^{7-9}$ Recently,

\footnotetext{
*Address for correspondence and reprint requests: Neil A. Zakai, MD, MSc, University of Vermont College of Medicine, 360 South Park Drive, Colchester, VT 05446; Telephone: 802-656-3154; Fax: 802-656-8965; E-mail: neil.zakai@ uvm.edu
}

Received: January 1, 2017; Revised: March 28, 2017;

Accepted: April 13, 2017

2017 Society of Hospital Medicine DOI: 10.12788/jhm.2831 the approval of direct oral anticoagulants (DOACs) by the Food and Drug Administration allows an all-oral treatment regimen for VTE, which could further facilitate outpatient treatment of DVT.

Costs associated with treatment of VTE are enormous. For outpatient treatment, researchers differ on individual estimates of cost savings associated with outpatient DVT management, but most report a cost savings of several thousand dollars per patient treated as an outpatient compared with as an inpatient. ${ }^{6,10}$ Given the incidence of DVT, reducing costs while maintaining a high quality of care in even a small percentage of DVT patients would result in significant healthcare cost savings as well as increased convenience for patients.

Despite high-quality evidence supporting the efficacy and safety of outpatient DVT treatment, little is known about the adoption of outpatient DVT treatment in the United States. Several studies that have been published were limited to single hospitals and were small in size $\mathrm{e}^{11,12}$ or limited to a cohort of patients already diagnosed with DVT. ${ }^{13}$

The purpose of this study was to report the frequency of outpatient treatment of DVT in the United States and describe patient characteristics associated with outpatient treatment. Information was gathered from The Reasons for 
Geographic and Racial Differences in Stroke (REGARDS) study, a contemporary cohort study of more than $30,000 \mathrm{pa}$ tients residing in the contiguous United States with racial and geographic diversity. We hypothesized that an individual's age, sex, race, region of residence, urban or rural residence, education level, and personal income would be associated with outpatient treatment. Results would allow the implementation of interventions to promote the appropriate use of outpatient treatment in order to reduce healthcare costs and increase patient convenience without compromising safety or efficacy of care.

\section{METHODS}

\section{Cohort Characteristics}

VTE events were ascertained in the REGARDS cohort, a prospective, longitudinal cohort study investigating the causes of racial and geographic disparities in stroke and cognitive decline. ${ }^{14}$ Between 2003 and 2007, there were 30,239 participants in the contiguous United States $\geq 45$ years old enrolled in REGARDS. By design, 55\% were female, $41 \%$ were black, the mean age was 65 years, and $56 \%$ lived in the southeastern United States. Participants were recruited from a commercial list by mail and telephone contact followed by verbal consent. A telephone interview was followed by an in-home examination, including obtaining written informed consent. On study entry, many participants had comorbid conditions, including $8 \%$ with reported atrial fibrillation, $56 \%$ receiving treatment for hypertension, $22 \%$ receiving treatment for diabetes, $3.7 \%$ taking warfarin, and $14 \%$ who were actively smoking. ${ }^{15,16}$ Participants were only excluded if they had active cancer, stated a self-reported race other than white or black, were unable to converse in English, had cognitive impairment as judged by the telephone interviewer, or were residing in or on the waiting list for a nursing home. Study methods were reviewed and approved by the institutional review boards at each study institution and have been published elsewhere. ${ }^{14}$

\section{Event Ascertainment and Definitions}

DVT event ascertainment is complete through 2011, with identification by telephone interview, review of reported hospitalizations, and review of deaths. ${ }^{17}$ Questionnaires in similar epidemiological studies have $98 \%$ specificity and $>70 \%$ sensitivity for ascertaining VTE events. ${ }^{18}$ A research nurse reviewed the text and recorded each reported hospitalization through 2011. Any report of a blood clot in the legs, arms, or lungs was a potential case for physician review. Medical records were retrieved for up to 1 year before and 1 year after potential events. Retrieved records were used to help guide further record retrieval if they did not contain the primary VTE event. Primary inpatient and outpatient records including history and physical examinations, discharge summaries, imaging reports (to include limb ultrasounds, computed tomography scans, and magnetic resonance imaging), autopsies, and outpatient notes were retrieved using up to 3 attempts. ${ }^{19}$ Using all available information, characteristics of the VTE event and treatment were systematically recorded. For each potential VTE case, two of three physician reviewers abstracted medical records to validate and classify the event. If the physician reviewers disagreed, the third physician would review the case, and if VTE status remained uncertain, cases were discussed and resolved. Race was determined by participant self-report as black or white. Location of residence was defined by geocoding the addresses, and urban or rural status was defined by United States census tract data using rural-urban commuting area codes (RUCA; with rural areas being RUCA codes $4-10) .{ }^{20}$ Other risk factors were obtained through surveys, telephone interviews, or in-home visits. ${ }^{14}$

Outpatient treatment was defined as receiving a DVT diagnosis in an emergency department or ambulatory clinic but not receiving an overnight hospitalization. Inpatient treatment was defined as at least 1 overnight stay in a hospital (but not in an emergency department). Only participants admitted with a primary diagnosis of DVT were included in the analysis. If someone was noted to have DVT but was admitted to the hospital for another cause, he or she was not included in the analysis and classified as a hospital-associated DVT. A provoked DVT was defined as occurring within 90 days of a major trauma, surgery, or marked immobility or was associated with active cancer or treatment for cancer (ie, chemotherapy, radiotherapy, or surgical therapy), while an unprovoked DVT was defined as having none of the above provoking factors. A distal DVT was defined as a DVT occurring in the posterior tibial, anterior tibial, peroneal, or soleus sinuses. The primary outcome was DVT treated as an outpatient only without concurrent diagnosis of PE or VTE as a complication of hospitalization (as these individuals were not eligible for outpatient treatment at the time).

\section{Statistical Analysis}

Age, sex, race, region of residence (inside or outside the southeastern United States), education, income (determined as greater or less than $\$ 20,000$ per year), and urban or rural status of residence were compared between DVT patients treated as outpatients and inpatients using $\chi 2$ analysis by inpatient or outpatient treatment. Univariable and multivariable logistic regression was then used to determine the odds ratio (OR) of receiving outpatient DVT treatment by the same variables with age per 10-year increment. ORs were adjusted for age, sex, race, year of DVT diagnosis, and region of residence as appropriate. Statistical significance was defined as $P<0.05$. All statistical analyses were performed by N.A.Z. and conducted with SAS version 9.3 (SAS Institute, Cary, NC). All authors had access to the primary clinical data.

\section{RESULTS}

Over a mean of 4.7 years follow-up, 379 VTE events occurred (incident and recurrent); 185 were diagnosed with a PE, and 53 occurred as a complication of hospitalization (and were not eligible for outpatient treatment), leaving 141 DVT events potentially eligible for outpatient treatment out 
TABLE 1. Characteristics of Participants with DVT by Treatment Location

\begin{tabular}{|c|c|c|c|}
\hline Characteristics & $\begin{array}{c}\text { Treated as Outpatient, } \\
\text { Total }=39\end{array}$ & $\begin{array}{l}\text { Treated as Inpatient, } \\
\text { Total }=102\end{array}$ & $P$ \\
\hline Median age (interquartile range) & 67 years $(60-73)$ & 70 years (63-76) & .02 \\
\hline DVT year (median) & 2009 & 2008 & .02 \\
\hline Female & $23(59 \%)$ & $43(42 \%)$ & .03 \\
\hline White & $30(77 \%)$ & $62(61 \%)$ & .04 \\
\hline Living outside the Southeast & $21(54 \%)$ & $50(49 \%)$ & .13 \\
\hline High school graduate & $38(97 \%)$ & $90(88 \%)$ & .07 \\
\hline Yearly income $>\$ 20,000$ & $12(82 \%)$ & $58(57 \%)$ & .02 \\
\hline Living in an urban area & $35(90 \%)$ & $71(70 \%)$ & .01 \\
\hline Provoked event & $10(26 \%)$ & $42(41 \%)$ & .09 \\
\hline Body mass index $\geq 30$ & $20(51 \%)$ & $47(47 \%)$ & .55 \\
\hline Current or former smoker & $17(44 \%)$ & $53(52 \%)$ & .45 \\
\hline Proximal DVT & $28(72 \%)$ & $92(90 \%)$ & $<.01$ \\
\hline Treated with full dose anticoagulation & $34(87 \%)$ & $89(87 \%)$ & .99 \\
\hline History of cancera & $6(25 \%)$ & $14(18 \%)$ & .43 \\
\hline Chronic kidney disease (eGFR <60) & $5(13 \%)$ & $19(19 \%)$ & .46 \\
\hline History of coronary artery diseasea & $8(21 \%)$ & $23(23 \%)$ & .77 \\
\hline History of hypertension & $21(54 \%)$ & $70(69 \%)$ & .10 \\
\hline History of diabetes ${ }^{*}$ & $4(11 \%)$ & $21(22 \%)$ & .15 \\
\hline History of hyperlipidemia* & $22(56 \%)$ & $53(54 \%)$ & .80 \\
\hline
\end{tabular}

aData were missing for 38 participants with cancer, 1 participant with coronary artery disease, 7 participants with diabetes, and 4 participants with hyperlipidemia.

NOTE: Abbreviations: DVT, deep vein thrombosis; eGFR, estimated glomerular filtration rate.

of a population of 29,556 participants with available records and follow-up in the cohort (Figure).

Of 141 DVT events, 39 (28\%) were treated as outpatients. Table 1 presents the characteristics of participants treated as inpatients and as outpatients. Factors significantly associated with outpatient DVT treatment were younger age, female sex, white race, residing in an urban area, having a distal DVT only, and having a higher income. In the study, DVT events were recorded between 2003 and 2011; the median year of a diagnosed DVT and treated as an outpatient was 2009, while the median year of inpatient treatment was 2008. Living in the Southeast versus the rest of the country $(P=0.13)$ and having a high school education or greater $(P$ $=0.07$ ) were marginally associated with receiving outpatient treatment. In absolute terms, $11 \%$ of people living in rural areas and $19 \%$ of black patients had outpatient DVT treatment while $33 \%$ of the urban dwellers and $32 \%$ of white patients received outpatient treatment (Table 1). At the time of cohort enrollment, $92 \%$ of participants claimed to have insurance; however, this did not differentiate between Medicare, Medicaid, and private insurance. Only 1 participant diagnosed with DVT had an estimated glomerular filtration rate $<30$, and this individual was admitted for treatment.

Table 2 reports the multivariable adjusted OR for outpa-

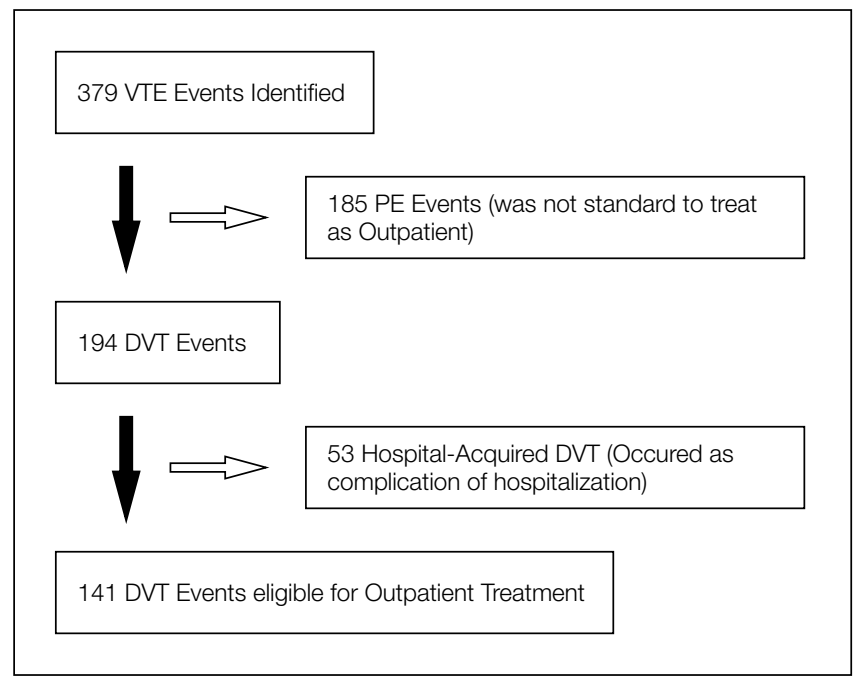

FIG. VTE Events in REGARDS

NOTE: Abbreviations: DVT, deep vein thrombosis; PE, pulmonary embolism; VTE, venous thromboembolism. 
TABLE 2. Likelihood of Receiving Outpatient Treatment for DVT

\begin{tabular}{ll}
\hline Variable & Adjusted Odds Ratio (95\% Cl) \\
\hline Age (per 10 years younger) & $1.90(1.19-3.02)$ \\
\hline Year of DVT diagnosis (per 1 year later) & $1.35(1.03-1.77)$ \\
\hline Sex (female versus male) & $2.41(1.06-5.47)$ \\
\hline Race (white versus black) & $3.29(1.30-8.30)$ \\
\hline Region (outside Southeast versus in Southeast) & $2.00(0.87-4.63)$ \\
\hline Education (high school graduate versus not) & $4.51(0.52-38.82)$ \\
\hline Income $>\$ 20,000$ (yes versus no) & $2.63(0.87-7.94)$ \\
\hline Living in an urban area (yes versus no) & $4.16(1.25-13.79)$ \\
\hline
\end{tabular}

${ }^{a}$ Adjusted for age, sex, race, VTE event year, and region.

NOTE: Abbreviations: $\mathrm{Cl}$, confidence interval; DVT, deep vein thrombosis; VTE, venous thromboembolism.

tient treatment of DVT adjusted for age, sex, race, region, and year of DVT diagnosis. Outpatient treatment of VTE was associated with younger age (OR 1.90; 95\% confidence interval [CI], 1.19-3.02 for every 10 years younger in age), female sex (OR 2.41; 95\% CI, 1.06-5.47), and white race (OR 3.29; 95\% CI, 1.30-8.30). For each progressive calendar year in which the diagnosis was made, individuals had a 1.35-fold increase in their odds (95\% CI, 1.03-1.77) of receiving outpatient treatment. Individuals living in urban areas were 4.16 (95\% CI, 1.25-13.79) times more likely to receive outpatient treatment than those in rural areas. Living outside of the southeastern United States and having an income of more than $\$ 20,000$ per year had increased, but nonsignificant, odds of being treated as outpatient (Table 2).

\section{DISCUSSION}

In this national, prospective, observational cohort study, only $28 \%$ of participants diagnosed with DVT were treated as outpatients versus being hospitalized. Urban area of residence, white race, female sex, and younger age were significantly associated with an increased odds of outpatient treatment. Groups that had particularly low outpatient treatment rates were rural dwellers and black participants, who had outpatient treatment rates of $11 \%$ and $19 \%$, respectively. The odds of receiving outpatient treatment did improve over the course of the study, but in the last year of VTE assessment, outpatient treatment remained at $40 \%$, but this was quite variable over the study years (being $8 \%$ two years prior).

The feasibility of outpatient treatment of DVTs requires a coordinated healthcare system and patient support to ensure education and appropriate anticoagulation monitoring. While not all DVTs should be treated as outpatients, differences in treatment location by sex, race, and residence point to potential healthcare disparities that increase the burden on patients and increase healthcare costs. Other studies have documented low outpatient treatment rates of
DVTs (20\% in 1 United States multicenter DVT registry) but have not discussed the associations of outpatient versus inpatient treatment. ${ }^{13}$ Outpatient treatment also appears to be underutilized in other developed countries; in the European Computerized Registry of Patients with Venous Thromboembolism, only $31 \%$ of DVT patients were treated on an outpatient basis between 2001 and 2011. ${ }^{21}$ To our knowledge, this is the first study to document the uptake of outpatient DVT treatment in the United States across multiple states, regions, and health systems well after the safety and efficacy of outpatient treatment of DVT was established by randomized controlled trials. ${ }^{3-5}$

The strengths of this study are that these data are derived from a contemporary cohort with a large geographic and racial distribution in the United States and are well characterized with a mean of 4.6 years follow-up. ${ }^{19}$ We are limited by a relatively small number of DVT events that were eligible for outpatient treatment $(n=141)$ and so may miss modest associations. Further, while the geographic scope of the cohort is a tremendous strength of our study, we may have missed some events and did not have complete record retrieval of reported events and could not assess access to healthcare in detail. These data were recorded before the use of DOACs became common. DOACs are an effective and safe alternative to conventional anticoagulation treatment for acute DVT. ${ }^{22}$ Their use might result in increased outpatient treatment, as they are not parenteral; however, cost considerations ( $\$ 400.00$ per month), especially with high-deductible insurance plans, may limit their impact on VTE treatment location. ${ }^{23}$ This study cannot account for why the racial, sex, and urban-rural differences exist, and by extension if hospitalization rates differ due to associated comorbidities or if this represents a healthcare disparity. While it is reasonable from a healthcare perspective that younger individuals would more likely be treated as outpatients, there is no data to suggest that differences in DVT by sex, race, and residential location support decreased outpatient treatment. Due to the age of the cohort, most individuals had some form of insurance and a primary care provider. However, we were unable to assess the quality of insurance and the ease of access to their primary care providers. More research is needed to determine whether patients were hospitalized on medical grounds or because of a lack of coordinated healthcare systems to care for them as outpatients.

In conclusion, only a minority of patients who were potentially eligible for outpatient DVT treatment (28\%) were treated as outpatients in this study, and there were significant racial and socioeconomic differences in who received inpatient and outpatient treatment. While outpatient treatment rates were below $40 \%$ in all groups, we identified groups with especially low likelihoods of receiving outpatient treatment. While all eligible individuals should be offered outpatient DVT treatment, these data highlight the need for specific efforts to overcome barriers to outpatient treatment in the elderly, rural areas, black patients, and men. Even modest increases in the rate of outpatient DVT treatment could result 
in substantial cost savings and increased patient convenience without compromising the efficacy or safety of medical care.

\section{Acknowledgements}

The authors thank the staff and participants of REGARDS for their important contributions. The executive committee of REGARDS reviewed and approved this manuscript for publication. This research project is supported by cooperative agreement U01 NS041588 from the National Institute of Neurological Disorders and Stroke, National Institutes of Health, Department of Health and Human Services. The content is solely the responsibility of the authors and does not necessarily represent the official views of the National Institute of Neurological Disorders and Stroke or the National Institutes of Health. Representatives of the funding agency

\section{References}

1. Raskob GE, Angchaisuksiri P, Blanco A N, et al. Thrombosis: A major contributor to global disease burden. Thromb Res. 2014;134(5):931-938. doi:10.1016/j.thromres.2014.08.014

2. Kearon C. Natural history of venous thromboembolism. Circulation. 2003;107(SUPPL. 23):22-31. doi:10.1161/01.CIR.0000078464.82671.78.

3. Koopman MM, Prandoni P, Piovella F, et al. Treatment of venous thrombosis with intravenous unfractionated heparin administered in the hospital as compared with subcutaneous low-molecular-weight heparin administered at home. The Tasman Study Group. N Engl J Med. 1996;334(11):682-687. doi:10.1056/ NEJM199603143341102.

4. Prandoni P, Lensing AW, Büller HR, et al. Comparison of subcutaneous low-molecular-weight heparin with intravenous standard heparin in proximal deep-vein thrombosis. Lancet. 1992;339(8791):441-445. doi:10.1016/S0196-0644(05)81047-9.

5. Levine M, Gent M, Hirsh J, et al. A comparison of low-molecular-weight heparin administered primarily at home with unfractionated heparin administered in the hospital for proximal deep-vein thrombosis. N Engl J Med. 1996;334(11):677-681. doi:10.1056/NEJM199603143341101

6. Segal JB, Bolger DT, Jenckes MW, et al. Outpatient therapy with low molecular weight heparin for the treatment of venous thromboembolism: a review of efficacy, safety, and costs. Am J Med. 2003;115(4):298-308. doi:10.1016/S0002 9343(03)00326-7.

7. Hyers TM, Agnelli G, Hull RD, et al. Antithrombotic therapy for venous throm boembolic disease. Chest. 2001;119(1 Suppl):176S-193S.

8. Hirsh J, Hoak J. Management of deep vein thrombosis and pulmonary embolism. A statement for healthcare professionals. Council on Thrombosis (in consultation with the Council on Cardiovascular Radiology), American Heart Association. Circulation. 1996;93(12):2212-2245.

9. Dunn AS, Coller B. Outpatient treatment of deep vein thrombosis: translating clinical trials into practice. Am J Med. 1999;106(6):660-669.

10. Spyropoulos AC, Lin J. Direct medical costs of venous thromboembolism and subsequent hospital readmission rates: an administrative claims analysis from 30 managed care organizations. J Manag Care Pharm. 2007;13(6):475-486. doi:2007(13)6: 475-486 [pii].

11. Lee M, Pao D, Hsu T, Sonderskov A. Cost savings and effectiveness of outpatient treatment with low molecular weight heparin of deep vein thrombosis in a community hospital. Can J Clin Pharmacol. 2004;11(1):e17-e27.

12. Pearson SD, Blair R, Halpert A, Eddy E, Mckean S. An outpatient program to have been involved in the review of the manuscript but not directly involved in the collection, management, analysis, or interpretation of the data. The authors thank the other investigators, the staff, and the participants of the REGARDS study for their valuable contributions. A full list of participating REGARDS investigators and institutions can be found at http://www.regardsstudy.org. Additional funding was provided by an investigator-initiated grant in aid from the American Recovery and Reinvestment Act grant RC1HL099460 from the National Heart, Lung, and Blood Institute. Work for the manuscript was supported in part by the Lake Champlain Cancer Research Organization (Burlington, Vermont).

Disclosure: The authors have no conflicts of interest to report

treat deep venous thrombosis with low-molecular-weight heparin. Eff Clin Pract. 1999;2(5):210-217.

13. Goldhaber SZ, Tapson VF. A prospective registry of 5,451 patients with ultrasound-confirmed deep vein thrombosis. Am J Cardiol. 2004;93(2):259-262. doi:10.1016/j.amjcard.2003.09.057.

14. Howard VJ, Cushman M, Pulley L, et al. The reasons for geographic and racial differences in stroke study: Objectives and design. Neuroepidemiology. 2005;25(3):135-143. doi:10.1159/000086678.

15. Meschia JF, Merrill P, Soliman EZ, et al. Racial disparities in awareness and treatment of atrial fibrillation: the REasons for Geographic and Racial Differences in Stroke (REGARDS) study. Stroke. 2010;41(4):581-587. doi:10.1161/STROKEAHA.109.573907.

16. Cushman M, Cantrell RA, McClure LA, et al. Estimated 10-year stroke risk by region and race in the United States: geographic and racial differences in stroke risk. Ann Neurol. 2008;64(5):507-513. doi:10.1002/ana.21493.

17. Wojcik NC, Huebner WW, Jorgensen G. Strategies for using the National Death Index and the Social Security Administration for death ascertainment in large occupational cohort mortality studies. Am J Epidemiol. 2010;172(4):469-477. doi:10.1093/aje/kwq130.

18. Frezzato M, Tosetto A, Rodeghiero F. Validated questionnaire for the identification of previous personal or familial venous thromboembolism. Am J Epidemiol. 1996;143(12):1257-1265

19. Zakai NA, McClure LA, Judd SE, et al. Racial and regional differences in venous thromboembolism in the United States in 3 cohorts. Circulation. 2014;129(14):1502-1509. doi:10.1161/CIRCULATIONAHA.113.006472.

20. Morrill R, Cromartie J, Hart G. Metropolitan, Urban, and Rural Commuting Areas: Toward a Better Depiction of the United States Settlement System. Urban Geogr. 1999;20(8):727-748. doi:10.2747/0272-3638.20.8.727.

21. Lozano F, Trujillo-Santos J, Barrón M, et al. Home versus in-hospital treatment of outpatients with acute deep venous thrombosis of the lower limbs. J Vasc Surg. 2014:59(5):1362-1367.e1. doi:10.1016/j.jvs.2013.11.091.

22. Robertson L, Kesteven P, McCaslin JE. Oral direct thrombin inhibitors or oral factor Xa inhibitors for the treatment of deep vein thrombosis. Cochrane Database Syst Rev. 2015;6:CD010956. doi:10.1002/14651858.CD010956.pub2.

23. Cushman M. Treating Acute Venous Thromboembolism - Shift with Care A New Era in the Treatment of Amyloidosis? N Engl J Med. 2013:29-30. doi:10.1056/NEJMe1307413. 